\title{
Comparative Analysis of Three Internal Fixation Methods for Intertrochanteric Fracture of Femur in the Elder
}

\author{
Junze Chen ${ }^{1, ~ *, ~ Y a n f e n ~ Z h e n g ', ~ Y o n g j i ~ X i n g ~}{ }^{3}$, Ye Lin ${ }^{3}$, Wen Zhong ${ }^{4}$ \\ ${ }^{1}$ Department of Orthopaedics, The People's Hospital of Baiyun District, Guangzhou, China \\ ${ }^{2}$ Department of Psychological Rehabilitation, Kunming Special Service Recuperation Center of the Army, Kunming, China \\ ${ }^{3}$ The PLA 74th Group Army Hospital, Guangzhou, China \\ ${ }^{4}$ The PLA 75th Group Army Hospital, Dali, China
}

Email address:

961262497@qq.com (Junze Chen)

${ }^{*}$ Corresponding author

\section{To cite this article:}

Junze Chen, Yanfen Zheng, Yongii Xing, Ye Lin, Wen Zhong. Comparative Analysis of Three Internal Fixation Methods for Intertrochanteric Fracture of Femur in the Elder. International Journal of Clinical and Experimental Medical Sciences. Vol. 6, No. 1, 2020 , pp. 5-11. doi: 10.11648/j.ijcems.20200601.12

Received: January 12, 2020; Accepted: February 24, 2020; Published: March 10, 2020

\begin{abstract}
As the average life span of the Chinese prolongs, the number of intertrochanteric fractures of the femur in elderly patients has increased all the time, and gradually becomes a more common type of fracture in elderly patients. From January 2011 to December 2016, 137 elderly patients with intertrochanteric fractures were treated with 3 mehhods, including locking plate, proximal femoral nail (PFNA) and artificial femoral head replacement. The mean age was $79.16 \pm 3.45$ years. The mean follow-up time was $17.20 \pm 1.30$ months. The operative time, intraoperative bleeding volume, postoperative complications, and Harris score of the hip joint at 1 month and 1 year after surgery were compared among the three groups. Statistical analysis by SPSS 22.0 software showed that the operation time of group $C$ was the shortest, followed by group B and group A. The difference was statistically significant $(\mathrm{p}<0.05)$. There was no significant difference in operation time between group A and group B $(\mathrm{p}>0.05)$; There was no significant difference in bleeding volume between the three groups $(\mathrm{p}>0.05)$. Group $\mathrm{A}$ had the lowest complication rate, followed by group $\mathrm{C}$ and group $\mathrm{B}$. But there was no significant difference between the three groups $\left(\mathrm{X}^{2}=0.0618, \mathrm{p}>0.05\right)$. All the patients were cured and discharged after active symptomatic treatment. Among the patients, the mean Harris score of group A was $38.52 \pm 6.51$ at 1-month follow up postoperatively; the mean Harris score of group B was 44.28 \pm 5.97 at 1-month follow up postoperatively; the mean Harris score of group C was 70.59 \pm 6.99 at 1-month follow up postoperatively, the $\mathrm{C}$ group was the best and the $\mathrm{B}$ group was the second. There was a significant difference between the three groups $(\mathrm{p}<0.05)$. The mean Harris score of group A was $84.40 \pm 9.34$ at 1-year follow up postoperatively; the mean Harris score of group B was 83.12 \pm 8.76 at 1-year follow up postoperatively; the mean Harris score of group C was 85.25 \pm 8.07 at 1-year follow up postoperatively. There was no significant difference between the three groups $(p>0.05)$. During the follow-up period, there was no loosening or breakage in the internal fixation, and the fracture healed well. This study concluded that locking plate, PFNA or artificial femoral head replacement can achieve satisfactory clinical results in elderly patients with intertrochanteric fractures, but artificial femoral head replacement can achieve early functional recovery, more conducive to elderly patients.
\end{abstract}

Keywords: Intertrochanteric Fractures, Locking Plate, Proximal Femoral Anti-rotation Intramedullary Nail, Artificial Femoral Head Replacement

\section{Introduction}

There are some possibilities in causing intertrochanteric fractures in the elderly due to direct and indirect violence injuries [1]. In recent years, with the prolongation of the average life span of the Chinese population, the number of elderly patients with intertrochanteric fractures has gradually increased. Intertrochanteric fractures of the femur can be occurred when the patient's hip is subjected to direct or indirect violent impact, or subjected to varus and forward 
stresses. Fractures occur under the pull of iliopsoas and gluteus medius muscles, followed by the separation of the greater trochanter and lesser trochanter [2, 3]. At present, surgical treatment is mainly used for this kind of disease. The purpose of active surgery is to reduce a series of complications for injuryed, bedridden elderly patients, to correct coxa vara, to restore the function of hip joint, to reduce pain and to greatly improve their quality of life [4]. There are many surgical treatments for intertrochanteric fractures in the elderly, among which locking plate [5], proximal femoral anti-rotation intramedullary nail (PFNA) [6] and artificial femoral head replacement [7] are the most widely used in the Guangzhou The people's Hospital of Baiyun District in the past five years. This study retrospectively analyzed 137 cases of intertrochanteric fractures of the femur in the elderly, and compared the clinical efficacy of three surgical methods in the treatment of intertrochanteric fractures of the femur.

\section{Methods}

\subsection{Study Design and Patients}

From January 2011 to December 2016, Table 1 shows that among the eligible patients, 58 were male and 79 were female, with a mean age of $79.16 \pm 3.45$ years and a mean course of disease of $17.20 \pm 1.30$ months. Type of internal fixation included Locking plate in 50 cases (Group A), PFNA in 45 cases (Group B) and artificial femoral head replacement in 42 cases (Group C). 3 patients in group A lost follow-up and 1 died naturally; 0 patients in group B lost follow-up and 0 died naturally; 1 patient in group $C$ lost follow-up and 1 died naturally (Table 1). AO classification distribution map of three groups of data (Figure 1).

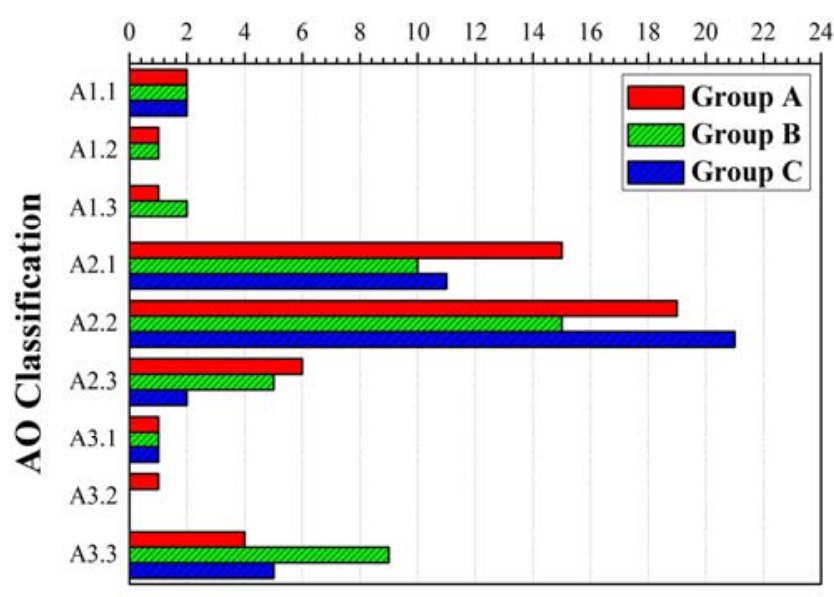

Number of Patients

Figure 1. AO classification distribution map of three groups of data.

Table 1. General data of eligible patients.

\begin{tabular}{ll}
\hline Variable & Value \\
\hline Age $(\mathrm{x} \pm \mathrm{s}$, year $)$ & $79.16 \pm 3.45$ \\
Sex $(\mathrm{n}, \%)$ & \\
Male & $58(42.34 \%)$ \\
Female & $79(57.66 \%)$ \\
Course of disease $(\mathrm{x} \pm \mathrm{s}$, month) & $17.20 \pm 1.30$ \\
\hline
\end{tabular}

\begin{tabular}{ll}
\hline Variable & Value \\
\hline Type of internal fixation & \\
Locking plate & $50(36.50 \%)$ \\
PFNA & $45(32.85 \%)$ \\
Artificial femoral head replacement & $42(30.65 \%)$ \\
\hline
\end{tabular}

\subsection{Inclusion Criteria}

Age $\geq 65$ years; simple intertrochanteric fracture of femur; no tumor disease; no severe osteoporosis or osteoporosis before effective treatment of bone mineral density $>-2.5$; no skin disease or a history of large doses of hormones; past life can be self-care; good limb movement function; patients and their families actively cooperate with surgical treatment, and agree to operate in accordance with medical ethical standards.

\subsection{Contraindications for Surgery}

According to anesthetist preoperative assessment of patient, the patient cannot tolerate the effect of anesthesia and surgery; combined with serious cardiovascular and cerebrovascular diseases; patients with blood system diseases [8], there is a contraindication of blood transfusion, it can not guarantee the smooth progress of intraoperative blood transfusion or postoperative blood supplement; suffering from respiratory diseases, it can not guarantee the stability of blood oxygen saturation; liver and kidney dysfunction. Often, the effect of medical treatment is not good; pressure sores or skin infections occur around the operation area $15-20 \mathrm{~cm}$; suffer from severe hypertension, diabetes, medical treatment is not good; long-term bedridden patients have lost activity before surgery; patients and their families refuse to surgical treatment.

\subsection{Preoperative Preparation}

In order to relieve pain after admission, patients can complete imaging examination under the protection of light weight traction brace, bedside skin traction and maintain the affected limb neutral position until the day of surgery [9]. Perfect laboratory blood tests: routine blood, urine and feces tests, liver and kidney functions, myocardial enzymes, electrolytes, blood sugar and other items. Monitoring the bone mineral density of patients, severe osteoporosis patients need symptomatic treatment of osteoporosis after improvement can be performed surgery [10]. For patients with hypertension, diabetes, old cerebral infarction and other diseases before consulting physicians to assist in diagnosis and treatment, until the underlying disease stabilized surgery [11].

\subsection{Surgical Methods}

\subsubsection{Operative Method of Locking Plate Group (Group A)}

After general anesthesia with endotracheal intubation, the patient lies on his back on the operating table, the buttocks are padded with anti-pressure sore pad, and the routine disinfection towel is spread. A longitudinal lateral incision is made from the apex of the greater trochanter of the femur to the proximal end of the thigh. The length is $12 \mathrm{~cm}$. The femur trochanter and proximal femur are exposed, the fracture end and the great and small trochanter are reduced, and 
temporarily fixed with Kirschner wire or steel The locking plate was placed on the outside of the proximal femur. The proximal end of the plate was transferred to 3-4 guide needles. During the operation, the fluoroscopy guide needle was drilled $1.5 \mathrm{~cm}$ below the femoral head. The depth of the nail passage was measured. The appropriate screw was selected for screw insertion. The distal end of the plate was screwed into each locking nail. The incision was sutured layer by layer, drainage tube was placed and the incision was sutured layer by layer.

\subsubsection{Operative Method of PFNA Group (Group B)}

After successful general anesthesia with tracheal intubation, the patient was lying on his back on the traction table, covered with a pressure ulcer pad under the buttocks. The fracture was reduced on the traction bed, and the fracture was reduced under C-arm fluoroscopy. After satisfactory reduction, the patient was fixed with a traction bed. Routine disinfection towel laying, centering on the apex of trochanter, makes a 6 $\mathrm{cm}$ surgical incision along the longitudinal axis of the extremity, exposes the apex of trochanter, takes the apex of trochanter as the point of insertion, opens the mouth, gradually enlarges the medullary cavity, guides the needle into the PFNA main nail, and inserts the Kirschner's needle after installing a sleeve at the proximal end of the main nail. Located in the center of the femoral head, about $1.5 \mathrm{~cm}$ below the femoral head, hammer into the screw blade after testing the depth of the screw path, screw in 2 distal screws, install the proximal tail cap, indwelling drainage tube, suture the surgical incision layer by layer.

\subsubsection{Operative Method of Artificial Hip Replacement Group (Group C)}

After successful general anesthesia with tracheal intubation, the patient was lying on the operating table, routinely disinfected and toweled, and the posterolateral incision of the hip joint was taken. The incision was made from 4 transverse fingers of the posterior superior iliac spine, and extended distally along the femoral axis through the apex of the greater trochanter. Incision of various layers of tissue to expose the femoral trochanter, gluteus medius muscle, cut short muscle group of external rotation, expose articular capsule, cut and switch segmental capsule, fully expose the fracture end, separate the distal and proximal end of the fracture, remove the proximal femoral head of the fracture, measure the diameter of the femoral head, clean up the surrounding bone fragments, reconstruct the stability of trochanter fracture with wire Kirschner wire, gradually use the medullary cavity enlarger. Expanding the medullary cavity until the medullary cavity matches the prosthesis sufficiently, choosing the appropriate artificial femoral stem prosthesis, and keeping the anteversion angle of 15 degrees, this study used the appropriate dual head. The stability of the joint was measured by reduction and movement of the joint, suture of the capsule and musculus brevis externus, indwelling drainage tube and suturing the incision layer by layer.

\subsection{Post-operative Management}

Group $\mathrm{A}$, at postoperative $2 \mathrm{~d}$, oral administration of
Rivaroxaban tablets 10mg, 1 times / d for 3 weeks. After $3 \mathrm{~d}$, under the protection of the brace, the injured limb did not carry the weight out of bed. 6-8 weeks after operation, most patients could remove braces and perform functional exercises on the affected hip joints. 3 months after surgery, further imaging examination showed that the fracture line at the fracture site was blurred, forming a bone connection, and the patient was instructed to walk with the affected limb.

Group B, 6-10h after hemostasis after operation, oral Rivaroxaban tablets $10 \mathrm{mg}, 1$ times / d for 3 weeks. After $3 \mathrm{~d}$, the patients were instructed to get out of bed, perform functional exercises of the hip joints, and prohibit loading. At 8-12 weeks after surgery, further imaging examination showed that the fracture line at the fracture site was blurred and the bone connection was formed, and the patient could walk with weight.

Group $\mathrm{C}$, after $7 \mathrm{~h}$, intravenous infusion of tranexamic acid 1g/d. 6-10h after hemostasis after operation, oral Rivaroxaban tablets $10 \mathrm{mg}, 1$ times / day, for 1 months. At postoperative $3 \mathrm{~d}$, the patient was able to get out of bed and walk with weight.

Statistical analysis

Continuous data was presented as mean \pm standard deviation (SD) and analyzed using the Student's $t$ test. Enumeration data were represented as per- centages, and the chi square test was used for comparison of the variables after operation. Statistical analysis was performed using SPSS 22.0. Two-sided $\mathrm{P}$ values $<0.05$ were considered significant.

\section{Results}

\subsection{Characteristics of the Patients}

137 patients were randomized to each group, 50 patients were treated by Locking plate in the group A. 45 patients were treated by PFNA in the group B, 42 patients were treated by hip replacement In the group $C$, there was no significant difference between three groups $\left(X^{2}=1.073, p>0.05\right)$. Among the eligible patients, 58 were male and 79 were female, there was a significant difference between male and female $\left(X^{2}=6.438, p<0.05\right)$. The mean age was $79.16 \pm 3.45$ years in this study, the mean age was $80.33 \pm 2.24$ years in group $A$, the mean age was $79.16 \pm 3.45$ years in group $B$, the mean age was $79.41 \pm 4.13$ years in group $C$, there was no significant difference between the three groups $\left(\mathrm{t}_{\mathrm{AB}}=2.177, \mathrm{t}_{\mathrm{AC}}=1.356\right.$, $\left.\mathrm{t}_{\mathrm{CB}}=0.301, \mathrm{p}>0.05\right)$. In this study, 131 cases were followed up at last with mean follow-up time $17.20 \pm 1.30$ months.

\subsection{Perioperative Period}

Each of the 137 patients completed the operation successfully. Among them, the mean operative time was $122.32 \pm 41.32$ in group A, $115.50 \pm 53.78$ in group B and $78.50 \pm 34.17$ in group $\mathrm{C}$. The operation time of group $\mathrm{C}$ was the shortest, followed by group B and group A. The difference was statistically significant $(p<0.05)$. There was no significant difference in operation time between group A and group B ( $p>0.05$ ); (Figure 2). The mean intraoperative blood loss was $310.25 \pm 68.25 \mathrm{ml}$ in group A, $295.50 \pm 75.84 \mathrm{ml}$ in group B and 
$330.60 \pm 87.92 \mathrm{ml}$ in group C. There was no significant difference in bleeding volume between the three groups $(\mathrm{p}>0.05)$. (Figure 3$)$

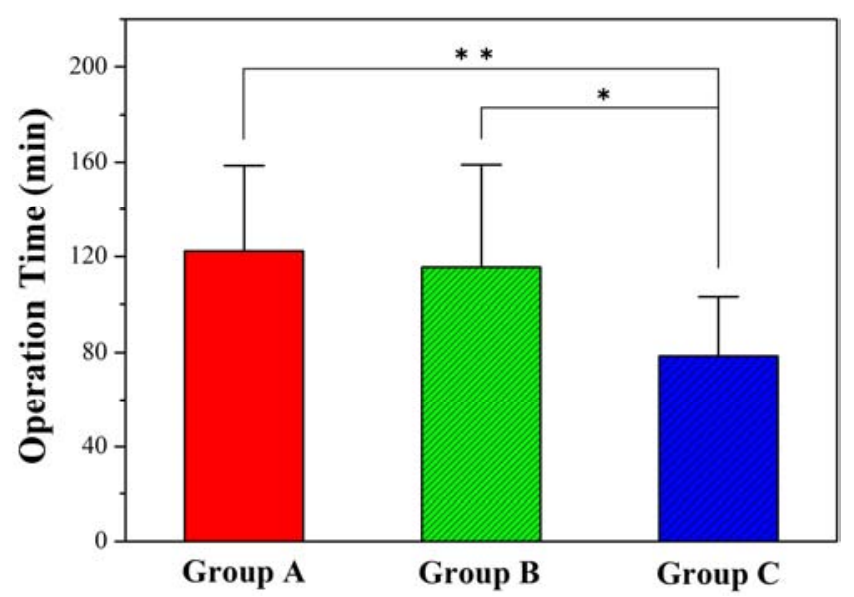

Figure 2. Operation time between group $A, B$ and $C$.

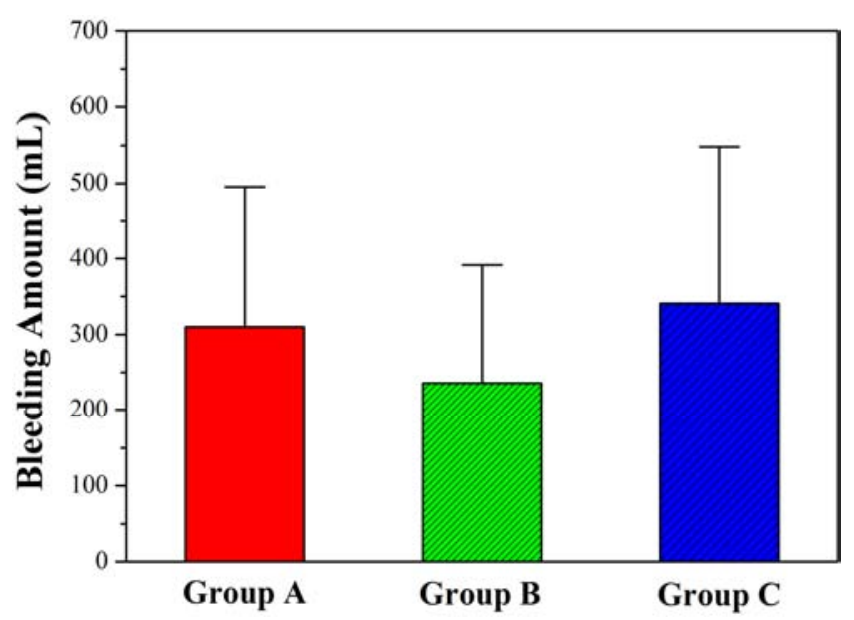

Figure 3. Bbleeding volume between group $A, B$ and $C$.

\subsection{Complications}

Complications included fracture of steel plate in 5 cases (35.71\%), fracture of screw in 3 cases $(21.43 \%)$, loosening of prosthesis in 4 cases (28. 57\%) and infection in 2 cases (14.29\%). Group A had the lowest complication rate, followed by $\mathrm{C}$ group and $\mathrm{B}$ group. But there was no significant difference between the three groups $\left(X^{2}=0.0618, p>0.05\right)$. All the patients were cured and discharged after active symptomatic treatment.

\subsection{Harris Score}

During the follow-up period, group A lost follow-up 3 people, 1 natural death; group B lost follow-up 0 people and 0 deaths; group $\mathrm{C}$ lost follow-up 1 person, 1 natural death. Harris score of group A in 46 cases, Harris score of group B in 45 cases and Harris score of group $C$ in 40 cases. Among the patients, the mean Harris score of group A was 38.52 \pm 6.51 at 1-month follow up postoperatively; the mean Harris score of group B was 44.28 \pm 5.97 at 1-month follow up postoperatively; the mean Harris score of group $C$ was $70.59 \pm 6.99$ at 1 -month follow up postoperatively, the $\mathrm{C}$ group was the best and the B group was the second. There was a significant difference between the three groups $(\mathrm{p}<0.05)$. The mean Harris score of group A was $84.40 \pm 9.34$ at 1-year follow up postoperatively; the mean Harris score of group B was 83.12 \pm 8.76 at 1-year follow up postoperatively; the mean Harris score of group $\mathrm{C}$ was $85.25 \pm 8.07$ at 1 -year follow up postoperatively. There was no significant difference between the three groups $(\mathrm{p}>0.05)$. (Figure 4$)$

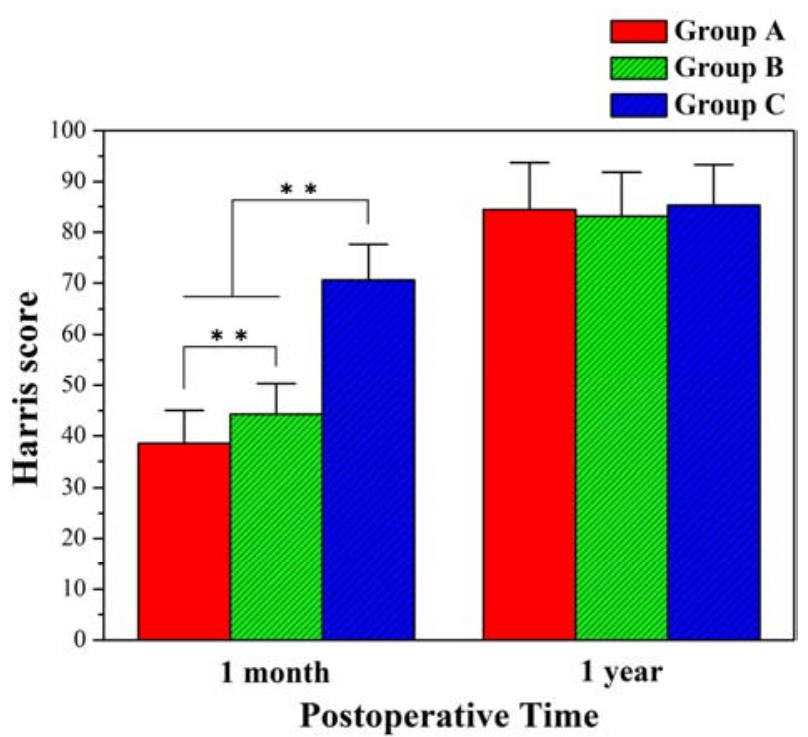

Figure 4. Harris score between the three groups.

\subsection{Surgical Outcomes}

Typical preoperative and postoperative X-rays of group A, female, 82 years old, comminuted intertrochanteric fracture of right femur (Figure 5 A-B). Typical preoperative and postoperative images of group B, female, 77 years old, comminuted intertrochanteric fracture of right femur (Figure 5 C-D). Typical preoperative and postoperative images of group $\mathrm{C}$, female, 79 years old, comminuted intertrochanteric fracture of right femur (Figure 5 E-F). During the follow-up period, there was no loosening or breakage in the internal fixation, and the fracture healed well (Figure 5).

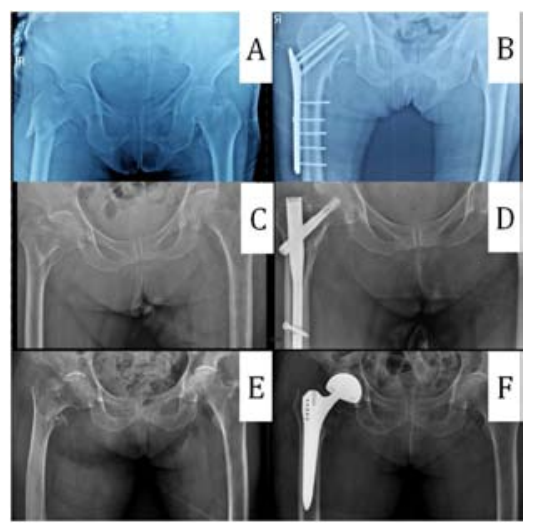

Figure 5. Preoperative and postoperative X-ray observation of three groups of internal fixation for intertrochanteric fractures in elderly patients. $X$-rays of group $A(A-B)$; X-rays of group $B(C-D) ; X$-rays of group $C(E-F)$. 


\section{Discussion}

Intertrochanteric fractures are common in the elderly over 65 years old [12]. In China, with the advent of an aging society, intertrochanteric fractures of the femur can occur in the elderly under low-energy injuries [13]. Therefore, intertrochanteric fractures of the femur in the elderly patients. The demand for surgical treatment is increasing. In this study, proximal femoral locking plate, PFNA, and artificial femoral head replacement was performed randomly selected for the treatment of intertrochanteric fractures in elderly patients over 65 years old. The operation time, intraoperative bleeding volume, postoperative complications and harris score of the hip joint were compared between the three groups.

Anatomical locking plate of proximal femur has a good bonding degree to the bone and a good tolerance to the trochanter, so that the plate covers the comminuted trochanter $[13,14]$. The multi-angle fixation with locking screws screwed through the locking hole at the proximal end of the plate has strong holding force and anti-rotation force, which is more suitable for the elderly patients with osteoporosis [5]. The steel plate has a common tension screw hole, which can be screwed into the tension screw to compress and fix the fractured end of the fracture, conducive to fracture healing. The locking screw-plate system does not need to be tightly bonded with the bone. It can reduce the stress shielding under the plate and reduce the blockage or damage of blood supply. It conforms to the BO fixation principle. But the locking plate is eccentric fixation, poor shear resistance, prone to fracture or failure of internal fixation risk, postoperative patients cannot carry out early weight bearing $[14,15]$. In this study, 50 elderly patients with intertrochanteric fractures of the femur were considered for locking fixation of comminuted fractures and protecting the blood supply of the fracture area. In this study, 45 patients were treated with PFNA. A spiral blade was inserted into the proximal femoral nail system to prevent rotation and squeeze the surrounding bone, thus increasing the bone density in the region. The internal fixation failure of DHS (Dynamic hip screw) and the risk of poor anti-rotation and fracture of Gamma nail were avoided. Because DHS is eccentric fixation and has long torque, compressive stress can not be transmitted through femoral moment when defect occurs in posteromedial cortex, which leads to significant increase of internal fixation stress and easy failure of internal fixation. Especially for inverse intertrochanteric fracture and serious osteoporotic unstable fracture, there are many complications $[4,16]$. Gamma nail has small force arm and short bending moment, which is superior to DSH $[6,17]$. Its single proximal tension screw makes Gamma nail have poor anti-rotation effect. The distal two interlocking nails have anti-fracture shortening and rotation effect, but they are prone to stress concentration, leading to femoral fracture at the end of the nail. In this study, 45 cases of unstable fractures were treated with PFNA. The operation time was short and the amount of bleeding was small. PFNA can be used for early mobilization and is suitable for most unstable fractures [18]. But intraoperative attention should be paid to. Benefit traction bed effective and maintain reset. The needle insertion point was located at the apex of the greater trochanter on the anteroposterior slice, and located on the anterior $1 / 3$ of the greater trochanter on the lateral film. Enter the needle direction: slightly inclined to the inside position, and the side needs to move forward slightly. Effective reduction of the posterior medial cortex can be achieved by open reduction if the reduction is not satisfactory. It is recommended to place a spiral blade in the center of the femoral head or below the femoral head. The insertion depth is $0.5-1.0 \mathrm{~cm}$ from the tip of the spiral blade to the surface of the femoral head. There is controversy about the possibility of using PFNA for lateral wall type and primary lateral wall rupture type $[6,19]$. The lateral wall refers to the lateral lateral cortex of the proximal femur far from the lateral femoral muscle crest. It is clinically classified into three types: Type I: lateral wall stabilized, equivalent to $\mathrm{AO}$ type $\mathrm{A} 1$, A2.1; Type II: lateral wall is dangerous, equivalent to AO type A2.2, A2.3; Type III: primary lateral wall fracture type, equivalent to AO type A3 type. Previous studies have found that PFNA fixation is effective in the treatment of comminuted greater trochanter fractures and associated with dangerous fractures of the lateral wall, but most believe that the risk of destroying the greater trochanter and the lateral wall increases $[11,12]$, which is a risk factor for internal fixation failure [20]. Proximal femoral locking plate is recommended. This study suggests that the proximal femoral locking plate is recommended for primary intertrochanteric fractures with ruptured lateral wall. There is no significant difference in operative time and bleeding volume between the two methods, but it can avoid aggravating the risk of damage to the greater trochanter and lateral wall. Whether artificial femoral head replacement is used in elderly patients with intertrochanteric fracture is controversial [21]. Femoral intertrochanteric fracture has abundant blood supply, few fracture nonunion occurs after operation, poor blood supply ability of elderly patients, body regeneration and repair ability is also reduced, self-resistance ability is reduced, artificial femoral head replacement can move to the ground as soon as possible after operation, early recovery; Fracture displacement caused by intertrochanteric fracture changes the normal anatomical relationship. Whether artificial femoral head replacement can satisfy all types of intertrochanteric fracture remains a risk of infection, dislocation and prosthesis loosening. Kaplan reported that there were no significant differences in postoperative complications, mortality and hip function recovery between intramedullary and extramedullary fixation, but the amount of bleeding and operation time were less than those of extramedullary fixation, and the recovery time of the affected hip was faster. Compared with PFNA internal fixation, PFNA had less operation time and bleeding volume, and had no significant difference in complications, but artificial femoral head replacement group could get out of bed early and recover joint function quickly. This study suggests that the use of artificial femoral head replacement for intertrochanteric fractures depends on the patient's age, osteoporosis, and the willingness of family members and patients. Older patients with osteoporosis in varying degrees, severe osteoporosis patients with internal fixation, there is a risk of internal fixation failure. 
Artificial joint replacement is divided into total hip replacement and artificial femoral head replacement. For patients aged 60-69 years, internal fixation is preferred. For patients aged 70-79 years, artificial femoral head replacement is recommended. Artificial joint replacement is recommended for patients over 80 years of age.

The choice of bone cements or biological prosthesis depends on the condition of osteoporosis. We tend to use bioprosthesis for acetabular prosthesis and bone cements prosthesis for femoral stem prosthesis.

For patients with severe osteoporosis intertrochanteric fractures, artificial joint replacement is also recommended. The operative skills and experience of this study are: Reconstruction of the greater trochanter is beneficial to the manipulation of the prosthesis, to the tension of gluteal and lateral femoral muscles, and to the stability of the prosthesis. For femur proximal bone defects, reconstruction of femoral moments is needed. Restore the original height of the greater trochanter through the joint prosthesis. Judging the anteversion angle through the shape of the trochanter or the proximal femoral medullary cavity after reduction.

The results of this study suggest that femoral head replacement can solve the problem of early getting out of bed for elderly patients, reduce the complications of bed rest and improve the quality of life of patients, and meet the needs of patients. The only shortcoming is the high cost. PFNA internal fixation is preferred if the lateral wall of femoral trochanter and PFNA entry point area are bony intact in elderly patients, and proximal femoral locking plate is preferred if there are comminuted fractures of lateral wall and greater trochanter.

\section{Conclusion}

Locking plate, PFNA or artificial femoral head replacement can achieve satisfactory clinical results in elderly patients with intertrochanteric fractures, but artificial femoral head replacement can achieve early functional recovery, more conducive to elderly patients.

\section{Competing Interests}

We have no competing interests.

\section{Research Ethics}

We received ethical approval from the ethic committee for examination and approval of Guangzhou The people's Hospital of Baiyun District to carry out our study. We received informed consent for the participants to participate in the study.

\section{References}

[1] Cooper, C., Cole, Z. A., Holroyd, C. R. et al. Secular trends in the incidence of hip and other osteoporotic fractures [J]. Osteoporos Int (2011) 22: 1277.
[2] Hui Z, Zeng X, Nan Z, et al. INTERTAN nail versus proximal femoral nail antirotation-Asia for intertrochanteric femur fractures in elderly patients with primary osteoporosis $[\mathrm{J}]$. Journal of International Medical Research, 2017, 45 (4): 300060517710584 .

[3] Banaszkiewicz P A. Traumatic Arthritis of the Hip After Dislocation and Acetabular Fractures: Treatment by Mold Arthroplasty: An End-Result Study Using a New Method of Result Evaluation [M]// Classic Papers in Orthopaedics. Springer London, 2014: 349-362.

[4] Kim J T, Kim H H, Kim J H, et al. Mid-Term Survivals After Cementless Bipolar Hemiarthroplasty for Unstable Intertrochanteric Fractures in Elderly Patients. [J]. Journal of Arthroplasty, 2017.

[5] Streubel P N, Moustoukas M J, Obremskey W T. Mechanical failure after locking plate fixation of unstable intertrochanteric femur fractures [J]. Journal of Orthopaedic Trauma, 2013, 27 (1): 22 .

[6] Aktselis, I., Kokoroghiannis, C., Fragkomichalos, E. et al. Prospective randomised controlled trial of an intramedullary nail versus a sliding hip screw for intertrochanteric fractures of the femur [J]. International Orthopaedics (SICOT) (2014) 38: 155 .

[7] C. Rogmark, O. Leonardsson; Hip arthroplasty for the treatment of displaced fractures of the femoral neck in elderly patients [J]. Bone Joint J 2016; 98-B: 291-7.

[8] Bakken, M. S., Engeland, A., Engesæter, L. B. et al. Risk of hip fracture among older people using anxiolytic and hypnotic drugs: a nationwide prospective cohort study [J]. Eur J Clin Pharmacol (2014) 70: 873.

[9] Neuman MD, Silber JH, Magaziner JS, Passarella MA, Mehta S, Werner RM. Survival and Functional Outcomes After Hip Fracture Among Nursing Home Residents. JAMA Intern Med. 2014; 174 (8): 1273-1280.

[10] Zimmerman M, Roberts K C, Brox W T. From Evidence to Application: AAOS Clinical Practice Guideline on Management of Hip Fractures in the Elderly. [J]. Journal of Orthopaedic Trauma, 2015, 29 (3): 119.

[11] Jacques Boddaert, Mathieu Raux, Frédéric Khiami, Bruno Riou; Perioperative Management of Elderly Patients with Hip Fracture. Anesthesiology 2014; 121 (6): 1336-1341. doi: 10.1097/ALN.0000000000000478.

[12] Roberts K C, Brox W T, Jevsevar D S, et al. Management of hip fractures in the elderly [J]. Journal of the American Academy of Orthopaedic Surgeons, 2015, 23 (2): 131.

[13] Li, J., Cheng, L. \& Jing, J. The Asia proximal femoral nail antirotation versus the standard proximal femoral antirotation nail for unstable intertrochanteric fractures in elderly Chinese patients. Orthopaedics \& traumatology, surgery \& research: OTSR 101, 143-146.

[14] Owsley, Kevin C, Gorczyca, et al. Displacement/Screw Cutout After Open Reduction and Locked Plate Fixation of Humeral Fractures [J]. Jbjs, 2008, 90 (2): 233-240.

[15] Zhang $\mathrm{H}$, Zhu X, Pei G, et al. A retrospective analysis of the InterTan nail and proximal femoral nail anti-rotation in the treatment of intertrochanteric fractures in elderly patients with osteoporosis: a minimum follow-up of 3 years [J]. Journal of Orthopaedic Surgery \& Research, 2017, 12 (1): 147. 
[16] Lems W F, Dreinhöfer K E, Bischoff-Ferrari H, et al. EULAR/EFORT recommendations for management of patients older than 50 years with a fragility fracture and prevention of subsequent fractures $[\mathrm{J}]$. Annals of the Rheumatic Diseases, 2017, 76 (5).

[17] Li M, Wu L, Liu Y, et al. Clinical evaluation of the Asian proximal femur intramedullary nail antirotation system (PFNA-II) for treatment of intertrochanteric fractures [J]. Journal of Orthopaedic Surgery \& Research, 2014, 9 (1): 112.

[18] Yu W, Zhang X, Zhu X, et al. Proximal femoral nails anti-rotation versus dynamic hip screws for treatment of stable intertrochanteric femur fractures: an outcome analyses with a minimum 4 years of follow-up [J]. Bmc Musculoskeletal Disorders, 2016, 17 (1): 1-6.
[19] Haidukewych GJ, Israel TA, Berry DJ. Reverse obliquity fractures of the intertrochanteric region of the femur. [J]. Journal of Bone and Joint Surgery-American Volume, 2001, 83-A (5): 643-650.

[20] Yang Y, Zhao X, Dong T, Yang Z, Zhang Q, Zhang Y (2017) Risk factors for postoperative delirium following hip fracture repair in elderly patients: a systematic review and meta-analysis. Aging Clin Exp Res 29: 115-126.

[21] K J Sheehan, L Williamson, J Alexander, C Filliter, B Sobolev, P Guy, L M Bearne, C Sackley; Prognostic factors of functional outcome after hip fracture surgery: a systematic review, Age and Ageing, Volume 47, Issue 5, 1 September 2018, Pages 661-670. 